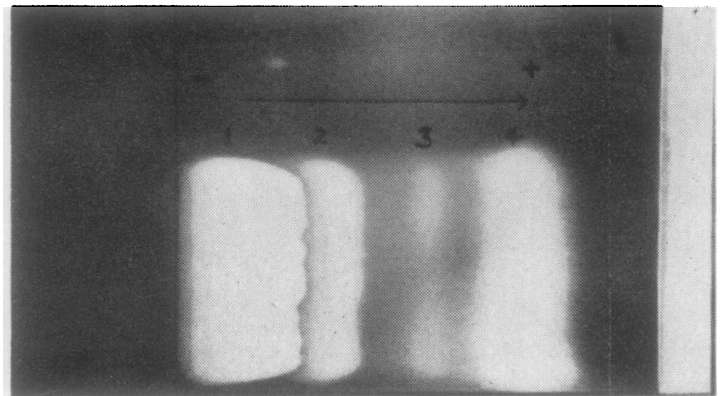

Fic. 1.-Urinary porphyrins photographed in ultra-violet light after paper electrophoresis from a case of porphyria.

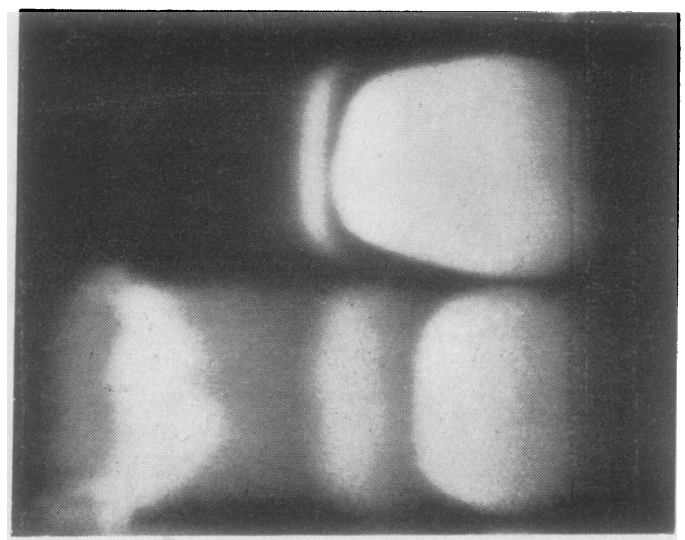

FIG. 2.-Pattern of porphyrin excretion in urine in two cases.

fluorescence filter giving ultra-violet light around 3,200 A.U. at 40 in. distance is used as the source. The separated porphyrin fractions show beautiful red fluorescence, and the area and the intensity of the fluorescence give an approximate relative proportion of the individual porphyrin fractions excreted in the urine (Figs. 1 and 2). For identification, purified porphyrins can be spotted as markers and run parallel with the porphyrin mixture extracted from the urine.

A characteristic electrophoretic pattern of porphyrin excretion can be obtained by this method and will be useful in comparing the porphyrin excretion in the same individual periodically, and also for studying the pattern of porphyrin excretion in cases of porphyrinuria and porphyria.

I am grateful to Dr. A. R. Natarajan, Chemical Examiner to the Government, and Mr. R. Viraraghavan for helping me to study and photograph the porphyrins in ultra-violet rays.

REFERENCE

Flynn, F. V., and Mayo, P. de (1951). Lancet, 2, 235.

\section{A Container for Handling and Centrifuging Pathological Material}

\section{B. FISHER AND A. J. HOGAN}

From the Department of Pathology and Bacteriology, $\overrightarrow{-}$ Welsh National School of Medicine, Cardiff

(RECEIVED FOR PUBLICATION MAY 17, 1957)

For many years workers in pathology laboratories $\vec{A}$ have used the "universal " container for the collection and handling of pathological material.

Although the "universal " container has proved to $N$ be a most useful bottle, it has the following serious drawbacks :

(1) Whitwell, Taylor, and Oliver (1957) found that 3 when infected fluid in a "universal " container is spun in an angle centrifuge some of the material is sprayed $\stackrel{\mathcal{S}}{\mathcal{O}}$ over a wide area around the centrifuge, the size of the $\vec{\theta}$ area being about $7 \mathrm{ft}$. in diameter.

If the containers are spun in a "swing-out" type of ${ }^{\infty}$ centrifuge the buckets and the outsides of the containers may be heavily contaminated, though neither the inside of the centrifuge casing nor the atmosphere is contaminated.

(2) Should at any time the cap be faulty or not $\frac{\mathscr{Q}}{\mathbb{Q}}$ properly tightened, material may escape and contami- $\varrho$ nate the outside of the bottle during normal handling.

(3) When material with a low cell content is centrifuged, cells are deposited across the whole of the floor of the bottle, and are then very difficult to recover.

In an attempt to overcome these faults, a bottle has been designed with a trough around the neck and an inner bottom surface which is conical (Fig. 1).

The bottle is made of transparent "bottle", glass, with a diameter of base $1 \frac{1}{8}$ in. and overall height of $3 \frac{1}{2}$ in. The neck is threaded to take the standard $1 \mathrm{oz}$. bottle metal cap with rubber liner, so as to allow the rim of the cap to come below the edge of the trough wall. The depth of the trough around the

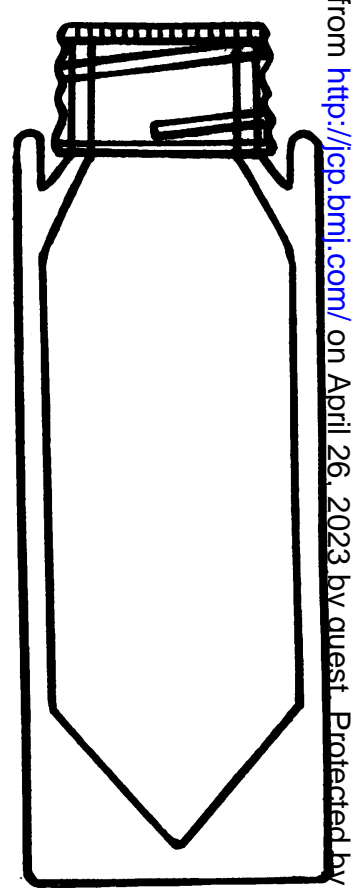

FIG. 1.-Narrow-neck containe? 


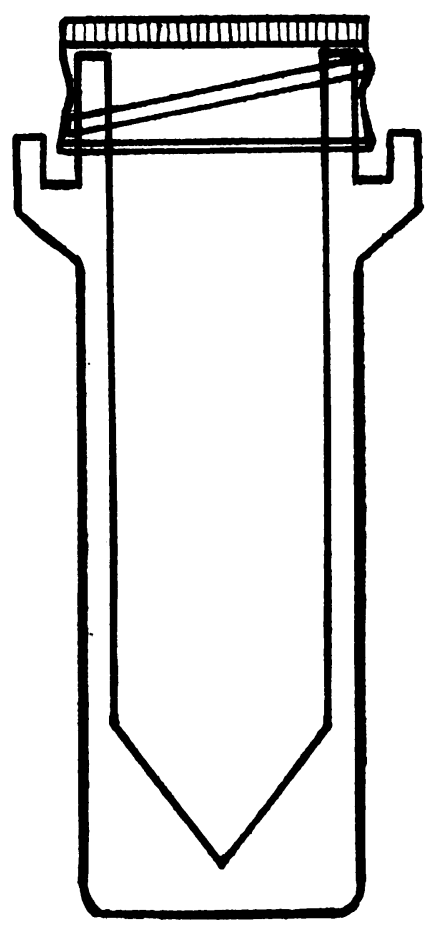

Fig. 2.-Wide-neck container.

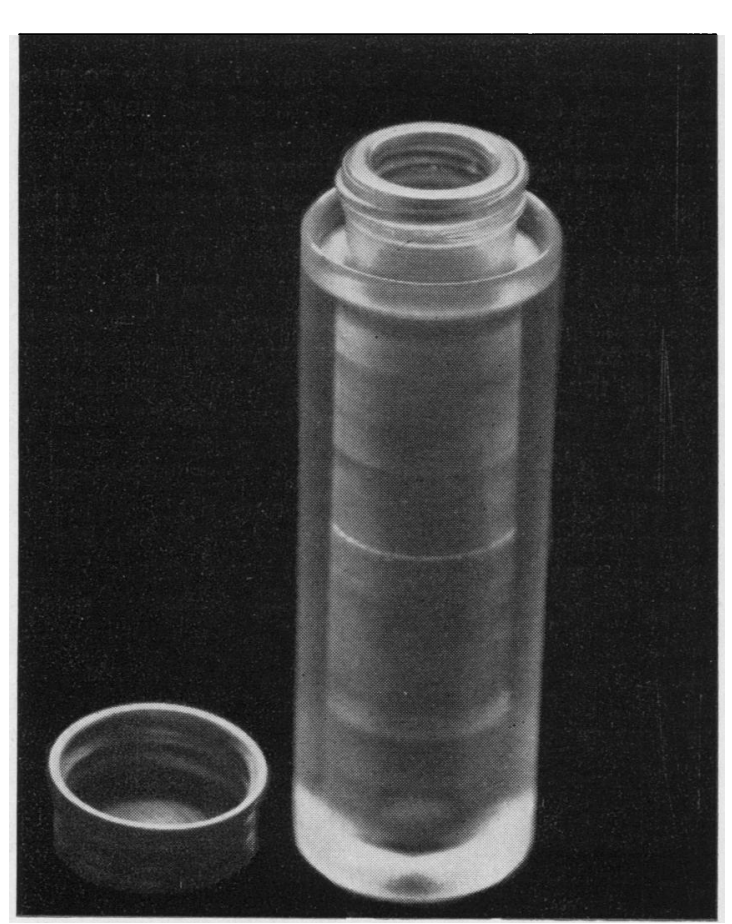

Fig. 3.-Perspex prototype bottle. neck is $3 / 16$ in., and the angle of floor from periphery to centre $45^{\circ}$.

The design may be modified so that the container has a wider neck and is threaded so that it will take a "universal" metal screw cap and liner (Fig. 2).

Owing to the difficulty in having a single container made in glass, it was decided to e m ploy perspex for the prototype bottle (Fig. 3). A 1 oz. narrow necked bottle was also made in perspex to use as a control in the tests.

to come in contact with the external surfaces of the necks of the bottles. About one third of the fluid was then poured out of the bottles and they were capped tightly with dry screw caps fitted with dry rubber liners.

The bottles were then wrapped in the filter paper impregnated with Andrade's indicator and centrifuged at 3,000 r.p.m. for three minutes in a "swing-out" type of centrifuge. On examination it was found that the filter paper surrounding the $1 \mathrm{oz}$. bottles was stained, indicating that hydrochloric acid had escaped. Examination of the paper surrounding the prototype container showed it to be unmarked, although a drop of fluid lying in the base of the trough could be seen.

The above tests were carried out several times in the "swing-out" type of centrifuge, and also in an angle centrifuge, always with a similar result.

A suspension of approximately 1,000 red blood cells per c.mm. was made in phy'siological saline, and $10 \mathrm{ml}$. of this fluid was placed in the prototype container, and also in a $1 \mathrm{oz}$. bottle. Both containers were centrifuged at 3,000 r.p.m. for three minutes and the red cell deposits examined. It was found that in the container with the conical bottom there was a compact "button" of cells which remained after the supernatant fluid had been carefully decanted. In the $1 \mathrm{oz}$. bottle the red cells were found to be distributed across the floor of the bottle with the majority of cells lying around the periphery of the floor, this being due to the slight convexity of the floor of the container. After carefully decanting the supernatant 
fluid the remaining cells were found to be intermixed with the residual supernatant fluid, and were very difficult to recover in a useful concentration.

\section{Summary}

A container has been described for the centrifuging and handling of pathological material.

The advantages of the container described are:

(1) Prevention of widespread contamination when centrifuging : (2) prevention of contamination of the outside of the container when pouring ; (3) prevention of contamination of the outside of the bottle by the fitting of a loose or faulty cap; (4) enabling a "button" to be formed when centrifuging in a "swing-out" type of centrifuge, thus saving the cost of conical centrifuge tubes.

We wish to thank Minerva (Cardiff), Ltd., for making the perspex containers.

REFERENCE

Whitwell, F., Taylor, P. J., and Oliver, A. J. (1957). J. clin. Path., 10, 88.

\section{BOOK REVIEW}

Disorders of the Blood, 8th ed. Sir Lionel Whitby and C. J. C. Britton. (Pp. viii $+878 ; 20$ colour plates and 125 figs. $£ 3$ 15s.). London: Churchill. 1957.

The eighth edition of "Whitby and Britton" finally appeared after the death of the senior author, and, although the galley proofs had been almost completed before he died, the final preparation of the eighth edition must have been an additional burden at an unfortunate time for Dr. Britton. The fact that there have been eight editions in 22 years not only shows that the popularity of the best-known English textbook of haematology is being maintained, but is also clear proof of the enormous growth of the subject during this time. Since the last edition in 1953 so much new work has appeared-and in fact so many new journals of haematology and allied subjectsthat the authors have had to add considerably to the subject-matter. This has been done effectively, and no fault can be found with the up-to-date information available, but the authors rightly point out that the book is now no longer a simple textbook on haematology but is really now a reference book for haematologists and haematological laboratories.

The authors, however, have not been quite so successful with the elimination of the old and now obsolete material, and this needs ruthless pruning in future issues, because it leads to disconnected chapters beginning with introductory material bearing no relationship to modern views and more recent work. The photomicrographs are also of variable standards, and many of the older ones could be omitted without loss, e.g., Fig. 39, p. 240 (which is no less than shocking), shows a macropolycyte which is much better seen in the colour Plate XI two pages previously. Fig. 10, which purports to be leucoblastic hyperplasia in a bone marrow section, is also quite meaningless. Some of the new black-and-white photomicrographs, $\mathrm{N}$ for example, Figs. 71 and 72 and Plate XIV, do $\Omega$ demonstrate what can be achieved by enlarged black- N and-white photographs aiming to show detail.

The book shows every sign of remaining the leader in its field and maintaining its well-deserved popularity. The references are more than adequate, and the price of $75 \mathrm{~s}$. compares most favourably with other publications of this size.

A. Gordon SignY. 